\title{
Performance Analysis of Multiple User Optical Code Division Multiple Access
}

\author{
Himanshu Monga*, R. S. Kaler \\ Department of Electronics and Communication Engineering, Thapar University, Patiala (Punjab), India \\ Email: "himanshumonga@gmail.com
}

Received October 21, 2014; revised November 23, 2014; accepted December 19, 2013

Copyright (C) 2014 Himanshu Monga, R. S. Kaler. This is an open access article distributed under the Creative Commons Attribution License, which permits unrestricted use, distribution, and reproduction in any medium, provided the original work is properly cited. In accordance of the Creative Commons Attribution License all Copyrights (C) 2014 are reserved for SCIRP and the owner of the intellectual property Himanshu Monga, R. S. Kaler. All Copyright (c) 2014 are guarded by law and by SCIRP as a guardian.

\begin{abstract}
In this paper, we discuss and analyze an optical code division multiple access for multiple user system. Media access control implementation has been considered. For fulfilling the huge need of bandwidth services, technology tends to move to optical networks and three major optical systems come into existence. Code division of the optical network is most used and real concept interacted with users up to this time. Optical code division multiple access provides complete spectrum to each and every user for the time of accessing the channel. In the paper, we have proposed network architecture with optical encoder and decoder along with optical code translator which is supporting multiple user systems. We integrate the code translator with encoding and decoding of optical code to use the optical network at full extent and present the simulation validation results of $6 \mathrm{~Gb} / \mathrm{s}$ 3-hop transmission by use of proposed architecture. Further, we have implemented experimentation with 6 users 3 Gb/s optical code division multiple access network. Through simulation structure, it is shown that the combination of encoding with EDFA in multiple user environment system provides improvement in bit error rate and also improves the multiple access interference. With increase of users in the network, MAI value increases and our proposed scheme controls interference in the network.
\end{abstract}

\section{KEYWORDS}

Optical Code Division Multiple Access; Strong Interference; Weak Interference; Bit Error Rate; Optical Encoding; Laser Diode Source; Multiple Access Interference

\section{Introduction}

Modern Communication is intended to achieve greater heights for providing best services to the users. Due to heavy demand of bandwidth on remote access sites, optical code division multiple access networks need to be improved. Erbium-doped fiber amplifier is very useful for providing various properties in optical communication as it provides higher level of compatibility with less loss with better gain while communication and also reduces noise parameters. EDFA process has been used for the cancellation of noise and to provide gain with couplers along with encoder and decoders in the network.

In this paper, optical networks are the main focus in context of optical code division multiple access technique

\footnotetext{
"Corresponding author.
}

based on optical networks. Optical networks face a lot of challenging tasks and issues which include the quality services to the users.

Code Division Multiplexed Access (CDMA) is a form of spread-spectrum, a family of digital communication techniques that have been used in military applications for many years. The core principle of spread-spectrum is the use of noise-like carrier waves, and bandwidth much wider than that required for simple point-to-point communication at the same data rate [1].

Optical code division multiple access (OCDMA) is one of the upcoming technologies for future multiple access networks along with WDMA and OTDMA. OCDMA is a spreading technique based on the concept of assigning codes to each user in a fiber optic communication network $[2,3]$. 
Optical code acquisition and synchronization plays a crucial role because the degradation in the performance of the system will be dramatic when the synchronization between receiver and transmitter is not ideal [4]. In optical CDMA, each user (or station) is assigned a unique binary unipolar signature sequence as its own address. Each user sends a signature sequence, corresponding to the address of its intended destination, for a data bit one, but transmits nothing for a data bit zero [5].

Codes for OCDMA have to be unipolar, orthogonal (minimum cross-correlation) and constant weight in nature to obtain low values of the bit error rate due to multiple access interference (MAI) [3].

The process of optical CDMA is described in section 1. The brief summary of analysis of OCDMA network and brief results have been done in this section. Further multiple user architecture and proposed work have been explained in Section 2. In last Section 3, results from experimentation and discussion have been explained.

\section{Multiple Hop \& Multiple User Architecture}

An OCDMA network can be viewed as a multiple-channel system, with each channel corresponding to a CDMA code $[6,7]$. Each OCDMA node is equipped with a transceiver for data transmission and detection, which encodes or decodes messages using the right code. Jaswinder et al. (2011) [8] provides the differential detection scheme which can reduce the multiple access interference in coherent and non-coherent for both synchronous and asynchronous optical code division multiple access networks with development of on-off keying concept. Keshavarzian et al (2005) [9] address the synchronization problem of an optical network using OOC codes of length $F$. The MS algorithm has two modes and an initializing part: first, $F$ different shifts (or cells) in the search space are partitioned into equal-sized groups each containing $M$ different shifts. Abbasali et al (2011) [5] provides the evaluation of Optical CDMA Systems by using the full utilization of optical encoding in presence of interference and receiver noises. The concept of optical encoding is shown in Figure 1 below.

From the study [11,12], we have developed a scheme which also controls the multiple access interference with help of modulation, encoding, EDFA and coupler for optical networks. The basic structure for the proposed scheme has been displayed in Figure 2 below.

As shown in Figure 2, the output of the optical encoder is applied to and $M \times M$ optical coupler. Based on proposed structure, probability of bit error rate and interference avoidance is provided in Equation (1) below.

\section{Results and Discussion}

The Concept of optical CDMA has been opted for experimentation and experimentation in this paper started with the implementation of Multiple User network with following parameters as explained in Table 1 below. Network Simulator version 2 is used for the experimentation of OCDMA network.

For the probability calculation of bit error rate for optical encoding process, we have considered variables selected randomly as $M^{E}$ and $M^{D}$ i.e. $Q_{M E M D}(i, j)$. Where $Q$ is representing probability factor for interference between two different codes for OCDMA system with weight $W$ and length $L$ is usually equal to $Q, Q$ and $1-3 Q$, respectively. Therefore $Q_{M E M D}(i, j)$ distribution along with parameters $X-1$ and $(Q, Q, 1-3 Q)$ are described by:

$$
\begin{aligned}
& Q_{M E M D}(i, j) \\
= & \frac{(X-1) !}{i ! j !(X-1-(i+j)) !} Q^{i+j}(1-3 Q)^{X-1-(i+j)}
\end{aligned}
$$

Where the approx. value of probability $Q$ has been provided for final solution.

The simulation scenario used for the experimentation is shown in Figure 3.

The performance of the OCDMA network has been judged through bit error rate and multiple access interference. Bit error rate is calculated for 30 users in Figure 4, for 60 users in Figure 5, for 120 users in Figure 6, for 240 users in Figure 7. Multiple access interference has shown in Figure 8. The proposed work provides variation with increase in the number of users in the network. The variation has been shown in term of graphs with bit error rate and access interference.

Implementation shows that the bit error rate increases as increase of users. Normal OCDMA network provides high bit error rate as compared to proposed OCDMA

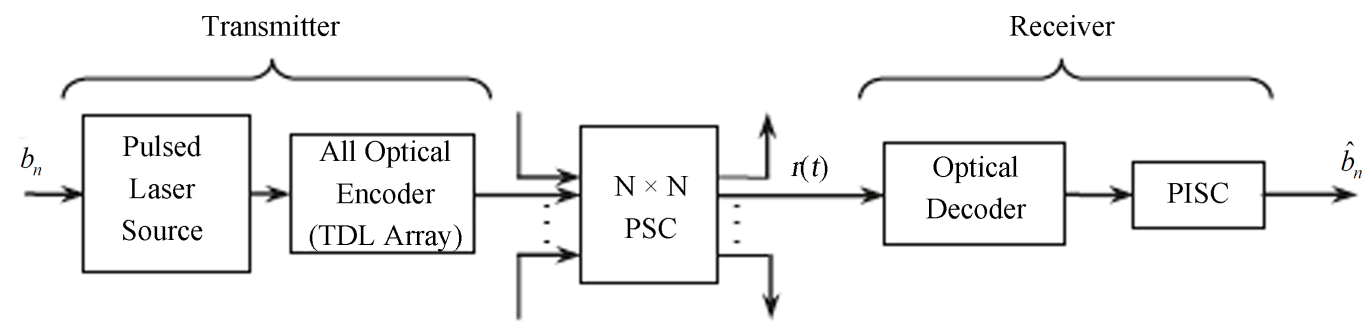

Figure 1. Model of OCDMA with Optical Encoding [10]. 


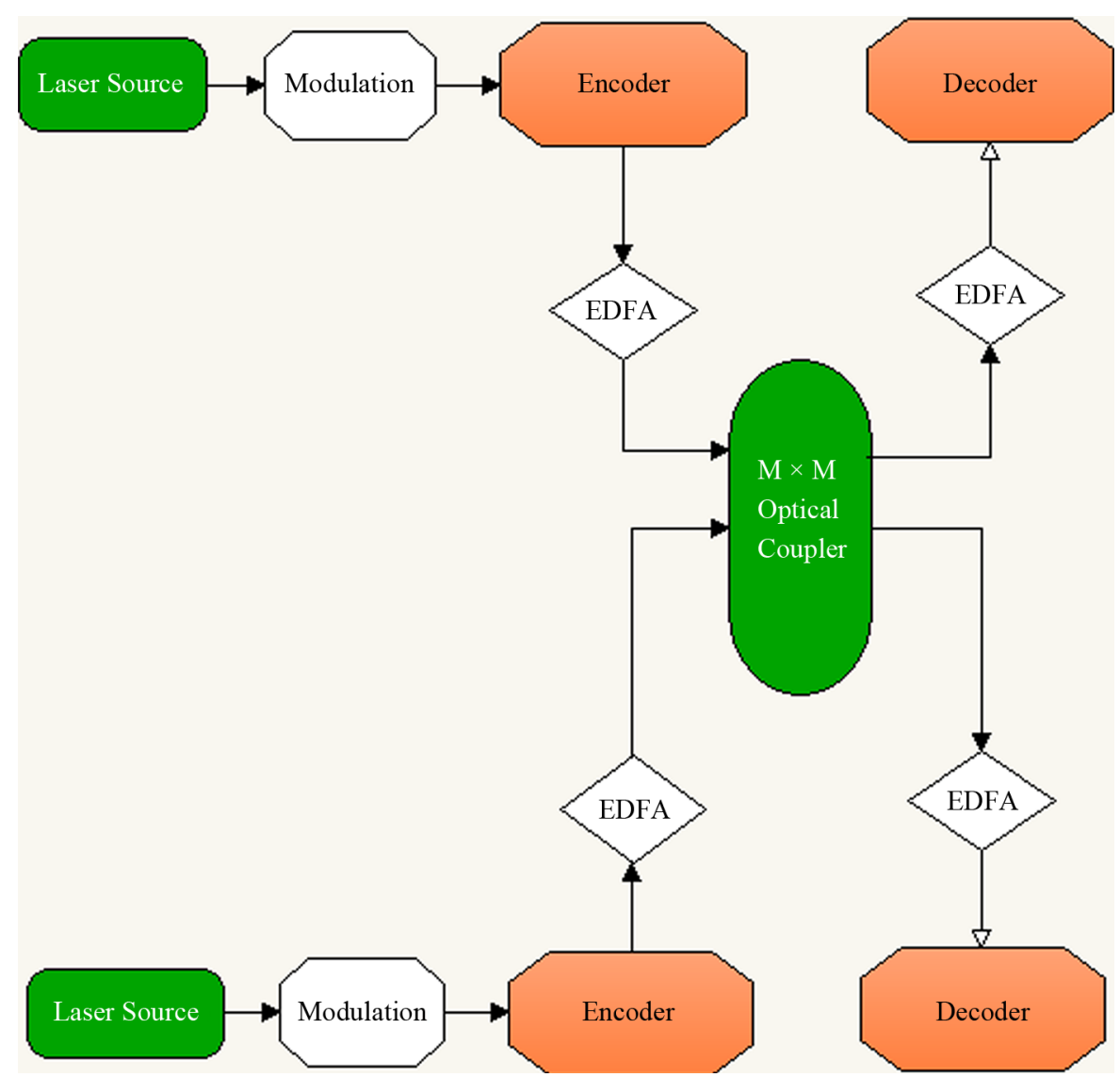

Figure 2. Node Architecture of Multiple User and Multi Hop concept.

Table 1. Parameters used in the simulation experimentation.

\begin{tabular}{cc}
\hline Parameter & Value \\
\hline Input power & $-3 \mathrm{dBm}$ \\
Data Rate & $6 \mathrm{Gbps}$ \\
Number of users & 6 \\
Length of Fiber & $20 \mathrm{~km}$ \\
Laser Frequency & $2.6244 \times 10^{14} \mathrm{~Hz}$ \\
Modulation Extinction Ratio & 100 \\
Dispersion & $15.75 \mathrm{ps} / \mathrm{nm}-\mathrm{km}$ \\
Attenuation of Fiber & $0.2 \mathrm{~dB} / \mathrm{km}$ \\
\hline
\end{tabular}

network which have low bit error rate comparatively. The initial state of the process carried slow interference cancelation which gave rise to the more bit error rate in starting seconds and after proper initiating of the proposed process bit error rate drops down and provides low error rate than normal Optical CDMA.

Implementation shows that the bit error rate increases as increase of users. Normal OCDMA network provides high bit error rate as compared to proposed OCDMA network which have low bit error rate comparatively. With increase of the users in the network, bit error rate goes high in normal Optical CDMA network as compared to proposed work. At peak intervals bit error rate provides similar results for some time and then normal network lacks cancelation of noise and suffers more error rate.

Implementation shows that the bit error rate increases as increase of users. Normal OCDMA network provides high bit error rate as compared to proposed OCDMA network which have low bit error rate comparatively.

Implementation shows that the bit error rate increases as increase of users. Normal OCDMA network provides high bit error rate as compared to proposed OCDMA network which have low bit error rate comparatively. With more number of users in the network, proposed structure provides low error rate and tends to normalize but the normal network tends to higher side.

The Multiple access interference value for proposed scheme is shown in Figure 8.

Figure 8 shows the high value of MAI for normal network as compared to the low value of MAI in proposed OCDMA. Approx. 10\% decrease in bot bit error rate and MAI proved to be good solution.

Overall consideration of the fact of growing need of the network, gave rise to the fast communication demand through optical networks. So OCDMA is one of the best solutions available for future communication. Proposed scheme for OCDMA provides much better results than 


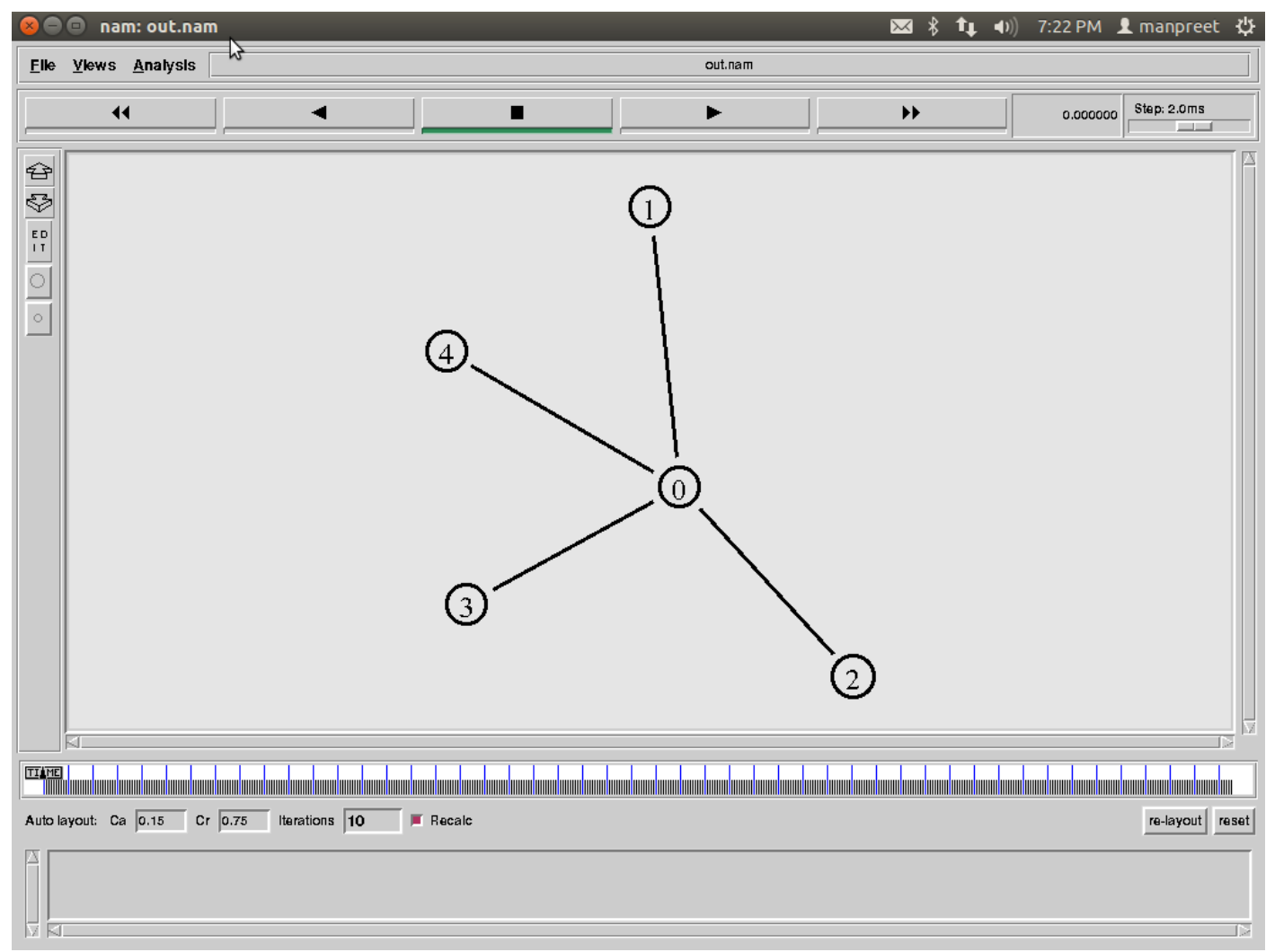

Figure 3. Overview of network simulation used for OCDMA network.

30 Users

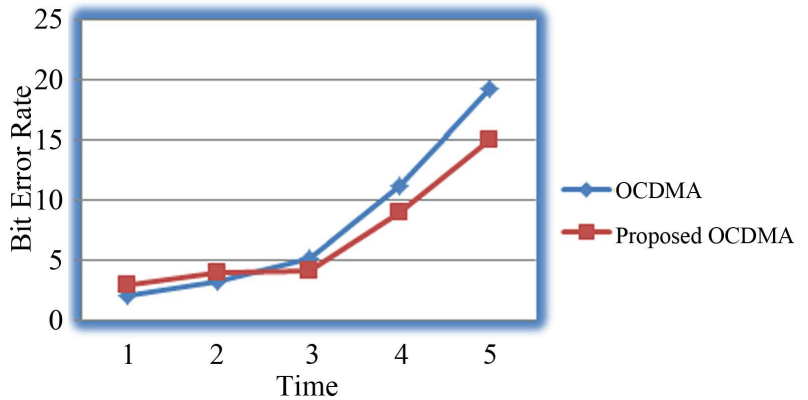

Figure 4. Bit Error Rate for proposed OCDMA with 30 users.

60 Users

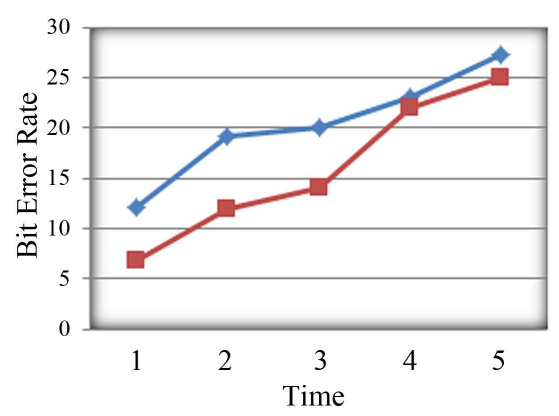

$\leadsto$ OCDMA

- - Proposed OCDMA

Figure 5. Bit Error Rate for proposed OCDMA with 60 users.
120 Users

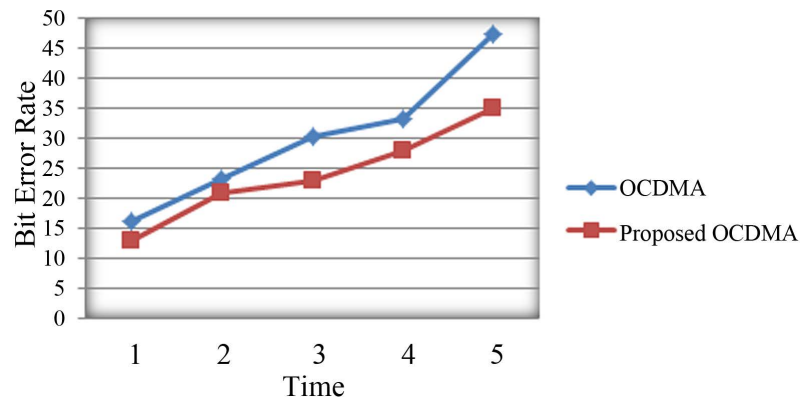

Figure 6. Bit Error Rate for proposed OCDMA with 120 users.

240 Users

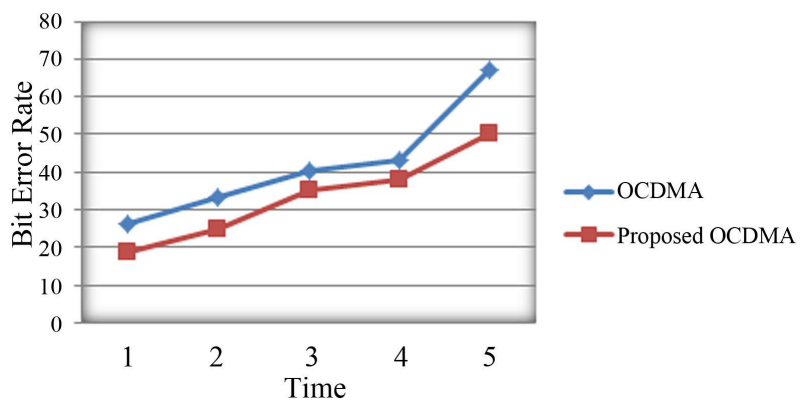

Figure 7. Bit Error Rate for proposed OCDMA with 240 users. 
30 Users

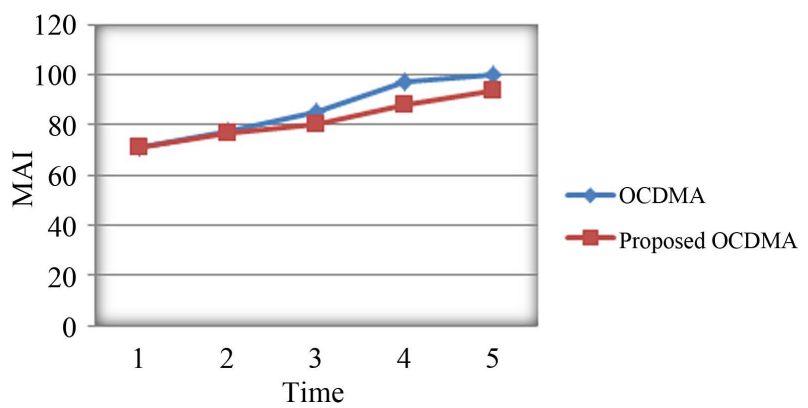

Figure 8. Multiple access interference for proposed and normal OCDMA.

the normal OCDMA network and proposed work also provides add on multiuser communication as compared to single user communication in normal OCDMA network.

\section{Conclusion}

This paper provides the idea of performance of multi user optical code division multiple access asynchronous networks with bit error rate and multiple access interference with the help of encoding process along with EDFA structure. Proposed scheme architecture provides less interference as compared to the normal optical code division multiple access networks. Multiple access interference is always been a limitation factor in optical CDMA networks and we have tried to refine the communication by decreasing the interference and bit error rate. Experimentation considers 6 users for the communication structure and shows similar results as suggested by interference cancellation technique with differential detection process.

\section{Acknowledgements}

We are very thankful for the contribution of Thapar University's technical department and Electronics Department for providing support for the experimentation.

\section{REFERENCES}

[1] C.-H. Lee, S. Zhong, X. Lin and Y. J. Chen, "Proposed Planar Light Wave Circuit Design of Programmable Optical CDMA Spectral Encoder/Decoder Using ArrayWaveguide Grating," IEEE Nanostructures and Quantum dots/RF photonics for CATV, San Diego, 26-30 July 1999, pp. 1139- 1140 .
[2] F. Benedetto, "On Efficient Code Acquisition of Optical Orthogonal Codes in Optical CDMA Systems,” IEEE Transactions on Communications, Vol. 58, No. 2, 2010, pp. 438-441.

http://dx.doi.org/10.1109/TCOMM.2010.02.080043

[3] T. Srinivas, "Simulation and Performance Analysis of OCDMA Systems Based on 2-D W/T Codes," IEEE International Conference on Advances in Systems and Measurements, Hyderabad, 16-18 December 2011, pp. 1-4.

[4] W. C. Kwong and G.-C. Yang, "Design of Multilength Optical Orthogonal Codes for Optical CDMA Multimedia Networks," IEEE Transactions on Communication, Vol. 50, No. 8, 2002, pp. 1258-1265 http://dx.doi.org/10.1109/TCOMM.2002.801499

[5] A. G. Sabbagh, "Performance Analysis of Optical CDMA Systems Utilizing Optical Encoding in Presence of Interference and Receiver Noises,” Journal of Optical Communications, Vol. 32, 2011, pp. 177-186.

[6] M. Ravi Kumar, "Design and Analysis of Three-Dimensional OCDMA Code Families," Optical Switching and Networking, Vol. 6, 2009, pp. 243-249. http://dx.doi.org/10.1016/j.osn.2009.08.002

[7] L. H. Xu and Q. Q. Fan, “A New Optical Code Translation Scheme for multi-hop Network and Simulation of Multi-user Asynchronous OCDMA System,” The 9th International Conference on Optical Communications and Networks, Nanjing, 24-27 October 2010.

[8] J. Singh, "Techniques for Reduction of Multiple Access Interference in Fiber-Optic CDMA Systems,” Journal of Engineering Research and Studies, Vol. 2, No. 2, 2011, pp. 7-10.

[9] A. Keshavarzian and J. A. Salehi, "Multiple-Shift Code Acquisition of Opticalorthogonal Codes in Optical CDMA Systems," IEEE Transactions on Communications, Vol. 53, No. 4, 2005, pp. 687-697. http://dx.doi.org/10.1109/TCOMM.2005.843456

[10] R. S. Kaler, A. Sharma and T. S. Kamal, “Approximate and Exact Small Signal Analysis for Single Mode Fiber near Zero Dispersion Wavelengths with Higher Order Dispersion," Fiber and Integrated Optics, Taylor and Francis, Vol. 21, 2002, pp. 391-415.

[11] Rajneesh Randhawa, S. Singh, J. S Sohal and R. S. Kaler "Wavelength Converter Using Semiconductor Optical Amplifier Mach-Zender interferometer based on XPM at $40 \mathrm{~Gb} / \mathrm{s}$ for future transport networks," Fiber and Integrated Optics, Vol. 28, No. 2, 2009, pp 154-169. http://dx.doi.org/10.1080/01468030802213637

[12] S. Khazraei and M. R. Pakravan, "Comparison of AIr and IEEE 802.11 with optical CDMA in Wireless Infrared Communication,” ICT 2008 International Conference on Telecommunications, St. Petersburg, 16-19 June 2008, pp. $1-5$. 